\section{Effect of a single intravitreal bevacizumab injection on different optical coherence tomographic patterns of diabetic macular oedema}

A Koytak1, M Altinisik', E Sogutlu Sari², O Artunay', JC Umurhan Akkan ${ }^{1}$ and $\mathrm{K}^{1}$ Tuncer $^{1}$
${ }^{1}$ Department of Ophthalmology, Faculty of Medicine, Bezmialem Vakif University, Istanbul, Turkey

${ }^{2}$ Department of Ophthalmology, Faculty of Medicine, Balikesir

University, Balikesir, Turkey

Correspondence: A Koytak, Department of Ophthalmology, Faculty of Medicine, Bezmialem Vakif University, Adnan Menderes Bulvari Fatih, Istanbul 34093, Turkey.

Tel: + 905054828065 ;

Fax: + 902126217580

E-mail: akoytak@

yahoo.com

Received: 28 October 2012 Accepted in revised form: 24 January 2013 Published online: 8 March 2013

\begin{abstract}
Purpose The aim of this study is to compare the therapeutic effect of a single intravitreal bevacizumab (IVB) injection in eyes with diabetic macular oedema (DMO) of different patterns determined by optical coherence tomography (OCT).

Methods Medical records of patients who had a single IVB injection for DMO were analysed retrospectively. Eyes with a clinically significant DMO and a central foveal thickness (CFT) of $250 \mu \mathrm{m}$ or more determined by OCT were included in the analysis. Best-corrected visual acuity (BCVA), CFT and total macular volume values before and after the injection were recorded. Eyes were divided into sponge-like diffuse retinal thickening (DRT), cystoid macular oedema (CMO) and serous retinal detachment (SRD) groups according to the morphology on OCT. Results A total of 92 eyes (42 with DRT, 31 with CMO and 19 with SRD) were included in the study. There was no statistically significant variation between three groups regarding the change in BCVA $(P=0.695)$. CMO and SRD groups had greater reductions in their mean CFT, and the amount of reduction showed statistically significant variation between three groups $(P=0.048)$. However, no statistically significant difference was found between groups in terms of percentage of change in CFT $(P=0.278)$.

Conclusion CMO and SRD subtypes are associated with a greater reduction in the CFT than the DRT subtype. Although the
\end{abstract}

change in BCVA was not significantly different between groups, the DRT group showed markedly better visual improvement in proportion to the decrease in CFT.

Eye (2013) 27, 716-721; doi:10.1038/eye.2013.17; published online 8 March 2013

Keywords: diabetic macular oedema; intravitreal bevacizumab; optical coherence tomography

Introduction

Macular oedema is a major cause of vision loss in patients with diabetic retinopathy. Its complex and multifactorial pathogenesis is not fully understood yet. What is apparent is that the multifactorial disruption of inner and outer blood-retinal barriers leads to abnormal inflow of fluid into the neurosensory retina exceeding the outflow, ending up with intraretinal and subretinal fluid accumulation. ${ }^{1-4}$

Besides making the objective diagnosis and quantitative evaluation of macular oedema possible, optical coherence tomography (OCT) offers the opportunity to assess in vivo histologic cross-sections of the healthy and diseased retina, leading to a better understanding of pathologic processes. 5,6

Three different morphologic subtypes of macular oedema were defined according to OCT: 7,8 Sponge-like diffuse retinal thickening (DRT), cystoid macular oedema (CMO) and serous retinal detachment (SRD). DRT, which is 
reflected as diffuse thickening of retina with reduced intraretinal reflectivity on OCT, is assumed to be caused by intracytoplasmic swelling of Müller cells in the outer plexiform layer. $\mathrm{CMO}$, on the other hand, is a result of the liquefaction necrosis of the Müller cells with formation of cystoid cavities after prolonged retinal oedema. SRD is defined as subfoveal accumulation of fluid within a well defined area of retinal detachment. ${ }^{7-11}$

Bevacizumab is a complete full-length humanized antibody that binds to all subtypes of vascular endothelial growth factor and its intravitreal administration has been proved in several studies to be effective in the treatment of diabetic macular oedema (DMO). However, only a few publications addressed the issue of why some eyes respond to this treatment better than others. Beside several properties in common, it is likely that each morphologic subtype of macular oedema has distinctive pathophysiologic aspects that could be responsible of different treatment responses.

The aim of this study is to compare therapeutic effects of a single intravitreal bevacizumab (IVB) injection in eyes with DMO of different patterns determined by OCT.

\section{Materials and methods}

In this study, medical records of patients who had a single intravitreal injection of bevacizumab for the treatment of DMO were analysed retrospectively. Eyes that had a clinically significant macular oedema (according to ETDRS study) ${ }^{12}$ and a central foveal thickness (CFT) of $250 \mu \mathrm{m}$ or more determined by OCT were included in the analysis, regardless of their diabetic retinopathy stage. If both eyes of the same patient met the inclusion criteria, only one eye was assigned randomly for the study.

All patients have had their macular OCT (Stratus OCT; Carl Zeiss Meditec AG, Jena, Germany) measurements before IVB injection. The exclusion criteria included ocular surgery or trauma, intravitreal or periocular injection of any drug or laser photocoagulation within 6 months before the injection; history of any previous vitreoretinal surgical procedure; presence of concomitant retinal pathologies and glaucoma, considerable media opacities interfering with the reliability of OCT imaging, evidence of vitreomacular traction or epiretinal membrane on OCT. Eyes that received additional treatments such as laser photocoagulation during the follow-up period were also excluded from the study.

This study was approved by the institutional review board of Bezmialem Vakif University Faculty of Medicine.

Intravitreal injections was performed in the operating room under aseptic conditions. Topical anaesthesia was achieved by the instillation of at least three drops of proparacaine hydrochloride $0.5 \%$ (Alcaine; Alcon Laboratories Inc., Fort Worth, TX, USA). Povidon iodine (5\%) was applied to the lids and eyelashes, and instilled in the conjunctiva before draping. $1.25 \mathrm{mg} / 0.05 \mathrm{ml}$ of bevacizumab (Altuzan; Roche Diagnostics $\mathrm{GmbH}$, Mannheim, Germany) was then injected using a 30 -gauge needle at $4 \mathrm{~mm}$ posterior to the limbus $(3.5 \mathrm{~mm}$ in pseudophakic eyes). Finally, a drop of povidon iodine $5 \%$ was instilled in the injection site. A rise in intraocular pressure that compromised optic disc perfusion was treated with anterior chamber paracentesis.

Best-corrected visual acuities (BCVA) with a Snellen chart, CFT and total macular volume (TMV) values assessed with OCT before and 4 weeks after the injection were recorded. Eyes were divided into DRT, CMO and SRD groups according to the assessment of macular oedema morphology on OCT. When more than one oedema pattern were observed, the eye was included into the group of obviously predominant pattern. In cases when more than one pattern was present and none of them was obviously predominant, the eye was not included in the study.

In order to evaluate variations in the therapeutic effect of IVB injection on three subgroups of DMO, differences between pre-injection and post-injection BCVA, CMT and TMV data, as well as percent changes in CMT and TMV values are compared.

BCVA measurements were converted to their logMAR (logarithm of the minimum angle of resolution) equivalents for statistical analysis. Data are summarized as mean \pm standard variation. Pre-injection and postinjection data within each group are compared using paired $t$-test. Independent samples $t$-test is used to compare data between groups. Pearson $\chi^{2}$ test was used for comparative analyses of categorical variables.

Variations among three groups were analysed using analysis of variance. A $P$-value $<0.05$ was considered to be statistically significant.

\section{Results}

A total of 92 eyes (42 with DRT, 31 with CMO and 19 with SRD) were included in the study. Baseline demographic and clinical properties of the patients in each group are summarized in Table 1. Three groups did not differ significantly in terms of mean age $(P=0.651)$.

Tables 2-4 summarize BCVA and OCT measurement data before the injection. Pre-injection mean BCVA did not differ significantly between groups $(P=0.353)$. The differences between mean pre-injection CFT and TMV values of three groups, however, were statistically significant $(P<0.001$ and $P=0.02$, respectively).

When pre-injection and post-injection data were compared within each group, increases in the BCVA were 
found statistically significant in DRT and CMO groups $(P=0.001$ and $P=0.003$, respectively), and not significant in SRD group $(P=0.064)$. Mean CFT values, on the other hand, were significantly reduced in all three groups $(P<0.001$ in SRDT and CMO groups, $P=0.002$ in SRD group). The reduction in TMV was significant only in eyes with $\mathrm{CMO}(P<0.001)$.

Table 1 Demographic and clinical properties of the study groups

\begin{tabular}{lccc}
\hline & \multicolumn{3}{c}{ Groups } \\
\cline { 2 - 4 } & $\begin{array}{c}\text { DRT } \\
(\mathrm{n}=42)\end{array}$ & $\begin{array}{c}\text { CMO } \\
(\mathrm{n}=31)\end{array}$ & $\begin{array}{c}\text { SRD } \\
(\mathrm{n}=19)\end{array}$ \\
\hline Age (mean \pm SD) & $57.21 \pm 8.22$ & $59.29 \pm 11.73$ & $58.95 \pm 10.99$ \\
Sex (male/female) & $15 / 27$ & $11 / 20$ & $8 / 11$ \\
Eye (right/left) & $25 / 17$ & $16 / 15$ & $9 / 10$ \\
DRP (NP/P) & $29 / 13$ & $21 / 10$ & $11 / 8$ \\
PRP (yes/no) & $23 / 19$ & $14 / 17$ & $14 / 5$ \\
MGL (yes/no) & $17 / 25$ & $12 / 19$ & $13 / 6$ \\
Lens (phakic/ & $28 / 14$ & $21 / 10$ & $11 / 8$ \\
pseudophakic) & & & \\
\hline
\end{tabular}

Abbreviations: $\mathrm{CMO}$, cystoid macular oedema; DRP, diabetic retinopathy; DRT, sponge-like diffuse retinal thickening; MGL, macular grid laser; $\mathrm{NP}$, non-proliferative; $\mathrm{P}$, proliferative; $\mathrm{PRP}$, panretinal laser photocoagulation; $\mathrm{SRD}$, serous retinal detachment.

Table 2 Pre-injection and post-injection measurements of BCVA (logMAR)

\begin{tabular}{lcccc}
\hline & $\begin{array}{c}\text { Pre-injection } \\
\text { BCVA }\end{array}$ & $\begin{array}{c}\text { Post-injection } \\
\text { BCVA }\end{array}$ & Change & P-value \\
\hline $\begin{array}{l}\text { DRT } \\
\text { (mean } \pm \text { SD) }\end{array}$ & $0.75 \pm 0.48$ & $0.61 \pm 0.46$ & $-0.14 \pm 0.26$ & $0.001^{\mathrm{a}}$ \\
$\begin{array}{l}\text { CMO } \\
\text { (mean } \pm \text { SD) }\end{array}$ & $0.67 \pm 0.47$ & $0.53 \pm 0.44$ & $-0.13 \pm 0.23$ & $0.003^{\mathrm{a}}$ \\
$\begin{array}{l}\text { SRD } \\
\text { (mean } \pm \text { SD) }\end{array}$ & $0.88 \pm 0.57$ & $0.79 \pm 0.60$ & $-0.09 \pm 0.19$ & $0.064^{\mathrm{a}}$ \\
$P$-value & $0.353^{\mathrm{b}}$ & $0.191^{\mathrm{b}}$ & $0.695^{\mathrm{b}}$ & \\
\hline
\end{tabular}

Abbreviations: $\mathrm{BCVA}$, best-corrected visual acuity; $\mathrm{CMO}$, cystoid macular oedema; DRT, sponge-like diffuse retinal thickening; SRD, serous retinal detachment.

aIndependent samples $T$-test.

${ }^{\mathrm{b}}$ Analysis of variance (ANOVA).
The mean BCVA, CFT and TMV values measured at the control visit (4 weeks after the injection) are displayed in Tables 2-4. No statistically significant difference was found between groups in terms of postoperative BCVA $(P=0.191)$. On the other hand, three groups showed significant variation in post-injection CFT and TMV ( $P=0.049$ and $P<0.001$, respectively).

The mean improvement in logMAR BCVA was $-0.14 \pm 0.26$ in DRT group, $-0.13 \pm 0.23$ in $\mathrm{CMO}$ group and $-0.09 \pm 0.19$ in SRD group. There was no statistically significant variation between three groups regarding the change in BCVA $(P=0.695$; Table 2$)$.

Mean change in CFT was $-54.31 \pm 80.99 \mu \mathrm{m}$ (a decrease of $13.53 \%$ ) in DRT group, $-111.26 \pm 131.54 \mu \mathrm{m}$ (a decrease of 20.84\%) in CMO group and $-119.79 \pm 147.99 \mu \mathrm{m}$ (a decrease of $21.75 \%$ ) in SRD group. CMO and SRD groups had greater reductions in their mean CFT, and the amount of reduction showed statistically significant variation between three groups $(P=0.048)$. However, no statistically significant difference was found between groups in terms of percentage of change in CFT $(P=0.278)$ (Table 3$)$.

Following a single injection, $28.6 \%(12 / 42)$ of the eyes in DRT group, $25.8 \%(8 / 31)$ of the CMO group and $21.1 \%$ $(4 / 19)$ of the SRD group had a CFT reduced to $<250 \mu \mathrm{m}$. No significant difference was found between groups with respect to the percentage of eyes achieving a CFT of $<250 \mu \mathrm{m}(P=0.825)$. In all, $50 \%$ of the eyes in DRT group, $41.9 \%$ of the CMO group and $31.6 \%$ of the SRD group had a post-injection CFT of $250-400 \mu \mathrm{m}$. The ratio of eyes having a CFT $>400 \mu \mathrm{m}$ was $21.4 \%$ in DRT group, $32.3 \%$ in CMO group and $47.4 \%$ in SRD group (Figure 1).

Besides changes in CFT, shifts in pathomorphology of the macular oedema were assessed in each subgroup. In DRT group, $28.6 \%(12 / 42)$ of the eyes became dry with no or minimal fluid, and $61.9 \%(26 / 42)$ retained the morphology of DRT, while $9.5 \%$ became CMO (4/42). In CMO group, on the other hand, $25.8 \%$ (8/31) of the eyes became dry, $61.3 \%$ (19/31) continued to have CMO and $12.9 \%(4 / 31)$ turned into having DRT. None of the eyes in DRT and CMO groups was found to have a SRD at the control visit. Besides $21.1 \%(4 / 19)$ that became dry in SRD group, $26.3 \%(5 / 19)$ and $10.5 \%(2 / 19)$ of the eyes

Table 3 Pre-injection and post-injection measurements of central foveal thickness

\begin{tabular}{lccccc}
\hline & Pre-injection CFT & Post-injection CFT & Change & Percent change & P-value \\
\hline DRT (mean \pm SD) & $366.98 \pm 121.45$ & $312.67 \pm 115.38$ & $-54.31 \pm 80.99$ & $-13.53 \pm 21.32$ & $<0.001^{\mathrm{a}}$ \\
CMO (mean \pm SD) & $463.32 \pm 120.69$ & $352.06 \pm 109.62$ & $-111.26 \pm 131.54$ & $-20.84 \pm 24.35$ & $<0.001^{\mathrm{a}}$ \\
SRD (mean \pm SD) & $515.05 \pm 165.83$ & $395.26 \pm 163.17$ & $1119.79 \pm 147.99$ & $-21.75 \pm 23.36$ & $0.002^{\mathrm{a}}$ \\
$P$-value & $<0.001^{\mathrm{b}}$ & $0.049^{\mathrm{b}}$ & $0.048^{\mathrm{b}}$ & $0.278^{\mathrm{b}}$ & \\
\hline
\end{tabular}

Abbreviations: CFT, central foveal thickness; CMO, cystoid macular oedema; DRT, sponge-like diffuse retinal thickening; SRD, serous retinal detachment.

${ }^{a}$ Independent samples $T$-test.

${ }^{\mathrm{b}}$ Analysis of variance (ANOVA). 
Table 4 Pre-injection and post-injection measurements of total macular volume

\begin{tabular}{lccccc}
\hline & Pre-injection TMV & Post-injection TMV & Change & Percent change & P-value \\
\hline DRT (mean \pm SD) & $9.35 \pm 2.04$ & $9.07 \pm 1.82$ & $-0.28 \pm 1.63$ & $-1.24 \pm 17.82$ & $0.268^{\mathrm{a}}$ \\
CMO (mean \pm SD) & $8.79 \pm 1.90$ & $7.88 \pm 1.56$ & $-0.91 \pm 1.28$ & $-9.48 \pm 12.85$ & $<0.001^{\mathrm{a}}$ \\
SRD (mean \pm SD) & $11.03 \pm 2.58$ & $10.11 \pm 2.54$ & $-0.92 \pm 1.96$ & $-7.21 \pm 17.23$ & $0.055^{\mathrm{a}}$ \\
$P$-value & $0.002^{\mathrm{b}}$ & $<0.001^{\mathrm{b}}$ & $0.172^{\mathrm{b}}$ & $0.089^{\mathrm{b}}$ & \\
\hline
\end{tabular}

Abbreviations: CMO, cystoid macular oedema; DRT, sponge-like diffuse retinal thickening; SRD, serous retinal detachment; TMV, total macular volume.

${ }^{\text {a }}$ Independent samples T-test.

${ }^{\mathrm{b}}$ Analysis of variance (ANOVA).

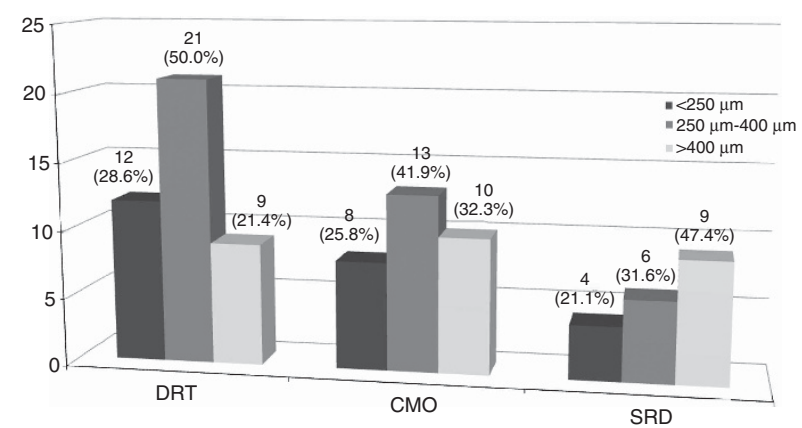

Figure 1 Numbers (percentages) of eyes in each group having a CFT below $250 \mu \mathrm{m}$, between 250 and $400 \mu \mathrm{m}$ and above $400 \mu \mathrm{m}$. $\mathrm{CMO}$, cystoid macular oedema; DRT, sponge-like diffuse retinal thickening; SRD, serous retinal detachment.

became having DRT and CMO, respectively, while $42.1 \%$ (8/19) remained with SRD.

The mean change in TMV was $-0.28 \pm 1.63 \mathrm{~mm}^{3}$ (a reduction of $1.24 \%$ ) in DRT group, $-0.91 \pm 1.28 \mathrm{~mm}^{3}$ (a reduction of $9.48 \%$ ) in CMO group and $-0.92 \pm$ $1.96 \mathrm{~mm}^{3}$ (a reduction of $7.21 \%$ ) in SRD group. Three groups did not differ in terms of the amount and percentage of reduction in TMV $(P=0.172$ and $P=0.089$, respectively; Table 4 ).

\section{Conclusion}

DRT, CMO and SRD are all parts of the clinical picture of diabetic retinopathy and it is possible that these morphologic patterns constitute episodes of the same pathophysiologic process. However, it is not irrational to argue that these subtypes have also their unique characteristics that influence their clinical courses, prognoses or treatment responses.

Kim et $a l^{13}$ have asked the question whether specific OCT patterns were predictive of visual outcome after focal laser photocoagulation in DMO. They have concluded that DRT pattern was associated with a greater reduction in retinal thickening and better visual acuity improvement compared with $\mathrm{CMO}$ pattern. Also, in a study by Lee and $\mathrm{Olk}^{14}$, eyes with CMO have been reported to respond poorly to modified grid laser. On the other hand, there is some degree of evidence that intravitreal triamcinolone acetonide (IVTA) worked better in eyes with CMO. Shulman et al ${ }^{15}$ have reported that visual improvement occurred in significantly more eyes with CMO than in those with DRT. Similarly, Shimura et $a l^{16}$ have found that IVTA was more effective in patients with $\mathrm{CMO}$ and less effective in those with SRD.

Several studies have proved the effectiveness of IVB in the treatment of DMO. However, only a few author have addressed the question of whether this treatment worked equally in all types of DMO. As in any other treatment option, it is undebatable that some eyes respond better to bevacizumab, while some do not improve at all. Identification of characteristics that influence the treatment response to bevacizumab would help to build up more effective management algorithms for different subtypes of DMO.

In a previously published study by Roh et $a l^{17}$ it is stated that patients showing CMO on OCT achieved greater improvements in visual acuity and macular thickness after IVB injection than patients with diffuse macular oedema. Conversely, Kim et al ${ }^{18}$ reported that the DRT type was associated with a greater reduction in the macular thickness and greater visual improvement than the CMO and SRD types.

In this retrospective study, we classified eyes with DMO into three groups according to their morphology on OCT and compared their response to a single intravitreal injection of bevacizumab in terms of changes in BCVA, CFT and TMV.

The DRT group, which is characterized by diffuse thickening of retina as a result of intracytoplasmic swelling of Müller cells, constituted $45.7 \%$ (42 eyes) of our study group. On the other hand, $\mathrm{CMO}$ characterized by cystoid cavities within the retina predominated in $33.7 \%$ (31 eyes) of the eyes. The remaining 20.7\% (19 eyes) of the eyes made up the SRD group, which is characterized by subfoveal accumulation of fluid as the distinctive part of clinical picture. Diffuse thickening of the retina was present in the majority of eyes along with $\mathrm{CMO}$ and/or SRD. Similarly, the coexistence of CMO and SRD was not a rare occurrence. Therefore, the composition of our study groups should not be 
interpreted as the actual distribution of oedema patterns in diabetic eyes. Rather, this should be regarded as an intentional classification aiming to emphasize the predominant morphology according to OCT crosssections. As there is not yet a commonly accepted classification system for DMO morphology, each author uses a different methodology to determine groups in studies with similar design. Nevertheless, in our study, DRT was the most common subtype of DMO, whereas SRD was the least common. This finding is consistent with most of the previous studies having worked with OCT patterns of DMO.7,8,13-18

In this study, three groups did not differ significantly in terms of mean baseline BCVA. Similarly, the change in BCVA in response to a single IVB injection was not significantly different among groups.

In contrast to BCVA, pre-injection CFT showed statistically significant difference between three groups and the mean CFT of DRT group was markedly lower than CMO and SRD groups. Also, the change in CFT was significantly smaller in DRT group than in CMO and SRD groups. However, when the increase in BCVA in proportion to the amount of decrease in CFT was analysed, the DRT group showed a better outcome. A ratio calculated as the mean increase in BCVA per a decrease of $100 \mu \mathrm{m}$ in mean CFT reveals this variation in the visual benefit from a single IVB injection. The DRT group had a BCVA improvement of $0.260 \log$ MAR per a decrease of $100 \mu \mathrm{m}$ in CFT. On the other hand, this ratio was 0.121 in the CMO group and 0.072 in the SRD group. This variation could be explained by different degrees of disruption of retinal morphology influencing the reversibility of visual deterioration in three subtypes of DMO. Therefore, the improvement in visual function should not be overlooked in favour of anatomic improvement while assessing treatment responses.

Another finding derived from this study is that some degree of transition was observed between DRT and CMO subtypes in response to a single injection of bevacizumab. On the other hand, although SRD became DRT or CMO in some eyes, none of the eyes in DRT and CMO groups was found to have a SRD at the control visit. This unidirectional shift of SRD to other subtypes along with a reduction in CFT implies that SRD could solely be a result of subretinal accumulation of excessive fluid originating from any type of oedema, rather than being a distinct morphologic subtype with a unique pathophysiology. However, our study on its own is far from providing sufficient data to make a definitive deduction about how SRD develops.

This study has several limitations that require consideration. Data were collected retrospectively from a relatively small number of patients. Also, eyes with different stages of retinopathy and variable visual potentials were included in our study. Thus, interpretations about the anatomic and visual outcomes are not totally free of bias. The purpose of this study is to assess the effect of a single IVB injection, which is accepted to appear and reach its maximum value during the first month. ${ }^{19}$ Therefore, the short follow-up time, which is 4 weeks, should not be considered as a shortcoming. Nevertheless, the continuity of treatment responses should be assessed with further studies with longer follow-up periods involving repetitive injections.

Our study shows, as a conclusion, that CMO and SRD subtypes are associated with a greater reduction in the CFT than the DRT subtype in response to a single IVB injection. Although the change in BCVA was not significantly different between groups, the DRT group showed a markedly better visual improvement in proportion to the decrease in CFT.

\section{Summary}

What was known before

- Three different morphologic subtypes of macular oedema were defined according to optical coherence tomographic patterns: sponge-like DRT, CMO and SRD. Several authors suggest that pathophysiologic mechanisms and treatment responses of these subtypes may be different. Several studies have proved the effectiveness of IVB in the treatment of DMO. However, only a few author have addressed the question of whether this treatment worked equally in all subtypes of DMO.

\section{What this study adds}

- The decrease in CFT in response to a single IVB injection was lower in DRT group. However, the improvement in visual acuity was not different between three groups.

\section{Conflict of interest}

The authors declare no conflict of interest.

\section{References}

1 Do Carmo A, Ramos P, Reis A, Proença R, Cunha-Vaz JG. Breakdown of the inner and outer blood retinal barrier in streptozotocin induced diabetes. Exp Eye Res 1998; 67: 569-175.

2 Sander B, Larsen M, Moldow B, Lund-Anderson H. Diabetic macular edema: passive and active transport of fluorescein through the blood-retina barrier. Invest Ophthalmol Vis Sci 2001; 42: 433-438.

3 Tso MO, Cunha-Vaz JG, Shih CY, Jones CW. Clinicopathologic study of blood-retinal barrier in experimental diabetes mellitus. Arch Ophthalmol 1980; 98: 2032-2040. 
4 Bhagat N, Grigorian RA, Tutela A, Zarbin MA. Diabetic macular edema: pathogenesis and treatment. Surv Ophthalmol 2009; 54: 1-32.

5 Hee MR, Puliafito CA, Wong C, Duker JS, Reichel E, Rutledge B et al. Quantitative assessment of macular edema with optical coherence tomography. Arch Ophthalmol 1995; 113: 1019-1029.

6 Hee MR, Puliafito CA, Duker JS, Reichel E, Coker JG, Wilkins JR et al. Topography of diabetic macular edema with optical coherence tomography. Ophthalmology 1998; 105 : 360-370.

7 Otani T, Kishi S, Maruyama Y. Patterns of diabetic macular edema with optical coherence tomography. Am J Ophthalmol 1999; 127: 688-693.

8 Kim BY, Smith SD, Kaiser PK. Optical coherence tomographic patterns of diabetic macular edema. Am J Ophthalmol 2006; 142: 405-412.

9 Yanoff M, Fine BS, Brucker AJ, Eagle Jr RC. Pathology of human cystoid macular edema. Surv Ophthalmol 1984; 28(Suppl): 505-511.

10 Fine BS, Brucker AJ. Macular edema and cystoid macular edema. Am J Ophthalmol 1981; 92: 466-481.

11 Tso MO. Pathology of cystoid macular edema. Ophthalmology 1982; 89: 902-915.

12 Early Treatment Diabetic Retinopathy Study Research Group. Photocoagulation for diabetic macular edema. Early treatment diabetic retinopathy study report number 1 . Arch Ophthalmol 1985; 103: 1796-1806.
13 Kim NR, Kim YJ, Chin HS, Moon YS. Optical coherence tomographic patterns in diabetic macular oedema: prediction of visual outcome after focal laser photocoagulation. Br J Ophthalmol 2009; 93: 901-905.

14 Lee CM, Olk RJ. Modified grid laser photocoagulation for diffuse diabetic macular edema. Long-term visual results. Ophthalmology 1991; 98: 1594-1602.

15 Shulman S, Ferencz JR, Gilady G, Ton Y, Assia E. Prognostic factors for visual acuity improvement after intravitreal triamcinolone injection. Eye 2007; 21: 1067-1070.

16 Shimura M, Yasuda K, Nakazawa T, Hirano Y, Sakamoto T, Ogura $Y$ et al. Visual outcome after intravitreal triamcinolone acetonide depends on optical coherence tomographic patterns in patients with diffuse diabetic macular edema. Retina 2011; 31: 748-754.

17 Roh MI, Kim JH, Kwon OW. Features of optical coherence tomography are predictive of visual outcomes after intravitreal bevacizumab injection for diabetic macular edema. Ophthalmologica 2010; 224: 374-380.

18 Kim M, Lee P, Kim Y, Yu SY, Kwak HW. Effect of intravitreal bevacizumab based on optical coherence tomography patterns of diabetic macular edema. Ophthalmologica 2011; 226: 138-144.

19 Arevalo JF, Sanchez JG, Wu L, Maia M, Alezzandrini AA, Brito $\mathrm{M}$ et al. Pan-American Collaborative Retina Study Group. Primary intravitreal bevacizumab for diffuse diabetic macular edema: the Pan-American Collaborative Retina Study Group at 24 months. Ophthalmology 2009; 116: 1488-1497. 